\title{
Tunable wavelength soft photoionization of ionic liquid vapors
}

Daniel Strasser ${ }^{1,5}$, Fabien Goulay, ${ }^{1,6}$, Leonid Belau ${ }^{2}$, Oleg Kostko $^{2}$, Christine Koh ${ }^{1}$, Steven D. Chambreau ${ }^{3}$, Ghanshyam L.Vaghjianit, Musahid Ahmed ${ }^{2}$ and Stephen R.

$$
\text { Leone } e^{1,2}
$$

${ }^{1}$ Departments of Chemistry and Physics, University of California, Berkeley, CA 94720, U.S.A.

${ }^{2}$ Chemical sciences division, Lawrence Berkeley National Laboratory, Berkeley, CA 94720, U.S.A.

${ }^{3}$ ERC, Incorporated, Edwards Air Force Base, CA 93524, U.S.A.

${ }^{4}$ Air Force Research Laboratory, Edwards Air Force Base, CA 93524, U.S.A.

${ }^{5}$ Present affiliation: Institute of Chemistry, Hebrew University, Jerusalem 91904, Israel.

${ }^{6}$ Present affiliation: Combustion Research Facility, Sandia National Laboratories, Livermore, CA 94551, U.S.A.

\begin{abstract}
$\underline{\text { Abstract }}$
Combined data of photoelectron spectra and photoionization efficiency curves in the near threshold ionization region of isolated ion-pairs from $[\mathrm{emim}]\left[\mathrm{Tf}_{2} \mathrm{~N}\right]$, $[\mathrm{emim}]\left[\mathrm{Pf}_{2} \mathrm{~N}\right]$ and $[\mathrm{dmpim}]\left[\mathrm{Tf}_{2} \mathrm{~N}\right]$ ionic liquid vapors reveal small shifts in the ionization energies of ion-pair systems due to cation and anion substitutions. Shifts towards higher binding energy following anion substitution are attributed to increased electronegativity of the anion itself, while shifts towards lower binding energies following cation substitution are attributed to an increase in the cation-anion distance that causes a lower Coulombic binding potential. The predominant ionization mechanism in the near threshold photon energy region is identified as dissociative ionization, involving dissociation of the ion-pair and the production of intact cations as the positively charged products.
\end{abstract}

Keywords: gas phase, ionic liquids, ionization energy, photoionization efficiency, photoelectron spectrum, synchrotron, high order harmonics. 


\section{Introduction}

Room temperature ionic liquids (ILs) are salts with a melting point near or below ambient temperatures. ${ }^{1}$ They are typically based on bulky multi-atom cations and/or anions that restrict the cation-anion distance of closest approach, thus limiting their Coulombic attraction and consequently also lowering their melting temperature even below room temperature. Melting temperatures of room temperature ILs can therefore be much lower compared to those of salts based on atomic ions, such as $\mathrm{NaCl}$, which has a melting temperature of $1074 \mathrm{~K}$. In addition to having low melting temperatures, ILs have a number of unique properties, including excellent solvation ability, extraordinarily low volatility, and high thermal stability. ${ }^{1-9}$ Since the physical and chemical properties of ILs can be varied by the choices of the cation and anion pair, ${ }^{1}$ they can be used for many applications such as fuel cells, ${ }^{3-5}$ batteries ${ }^{6,7}$ and solar cells. ${ }^{8,9}$ Furthermore, ILs are also used in ionic propulsion, ${ }^{10,11}$ for hypergolic fuels ${ }^{12}$ and various chemical extraction applications. ${ }^{13}$ The vast number of possible anion-cation combinations ${ }^{14}$ makes it valuable to rationally predict IL properties based on their constituents, ${ }^{15}$ and considerable experimental and theoretical work has been performed to predict and measure ionic liquid properties. ${ }^{16} \mathrm{Ab}$ initio calculations, used to predict the structure and interactions of ILs, are typically performed on isolated "gas phase" systems. ${ }^{17,18}$ It is therefore attractive to study isolated ion-pairs and to examine basic IL decomposition pathways, such as dissociation or ionization. Since ILs are generally perceived as "green solvents", their decomposition pathways can lead to a variety of products that need to be considered in specific applications. Given recent works ${ }^{19}$ demonstrating the distillation of selected families of ILs, we subsequently prepared molecular beams of IL vapor, providing a gas phase target of isolated ion pair species to obtain the photoelectron (PE) spectrum of $\left[\right.$ emim] $\left[\mathrm{Tf}_{2} \mathrm{~N}\right]$ vapor. $^{20}$ The direct comparison of our $[\mathrm{emim}]\left[\mathrm{Tf}_{2} \mathrm{~N}\right]$ vapor PE spectrum with previously measured PE spectra of a $[\mathrm{bmim}]\left[\mathrm{Tf}_{2} \mathrm{~N}\right]$ liquid surface, ${ }^{21,22}$ provided important evidence for the ion-pair nature of ionic liquid vapor. ${ }^{20}$ Other recent studies 
aimed at investigating the properties of IL vapor also include line of sight mass spectroscopy, ${ }^{23,24}$ IR spectroscopy,${ }^{25}$ and matrix isolated FTIR spectroscopy. ${ }^{26}$ Moreover, mass spectra of cation and anion substituted $\mathrm{ILs}^{20}$ showed that the ionization of these isolated IL ion-pairs by absorption of a single $23.2 \mathrm{eV}$ photon, which is more than $14 \mathrm{eV}$ above the ionization threshold, results in fragmentation with a predominant product of the intact cations. Interestingly, no signal is detected for the ionized parent ion-pair mass - i.e. removal of an electron from the ion-pair system results in dissociation of the ion pair. Since those results were obtained with a photon energy that is $14.3 \mathrm{eV}$ higher than the energy needed to remove an electron from the ion-pair, which could provide ample energy to detach an electron as well dissociate the molecule, near threshold investigations are required. Field induced IL ionization studies recently provide evidence for the possible formation of undissociated parent ion systems with the combined mass of the cation and anion. ${ }^{27}$

Here we present a study of the near threshold soft photoionization of isolated ion pairs with tunable photon energies between $8.3 \mathrm{eV}$ and $9.5 \mathrm{eV}$. Electronic structure changes following cation and anion substitutions are presented and discussed in terms of the observed binding energy shifts. Starting from the commonly used [1-ethyl-3methylimidazolium][bis(trifluoromethylsulfonyl)imide) $\quad\left(\left[\mathrm{emim}^{2}\left[\mathrm{Tf}_{2} \mathrm{~N}\right]\right) \quad\right.$ room temperature IL, shown in Fig. 1, the $[\mathrm{emim}]^{+}$cation is substituted by the bulkier imidazolium based 1,2-dimethyl-3-propylimidazolium ion $\left([\mathrm{dmpim}]^{+}\right)$, and the $\left[\mathrm{Tf}_{2} \mathrm{~N}\right]^{-}$ anion is substituted by the bis(pentafluoroethylsulfonyl)imide ion $\left.\left(\left[\mathrm{Pf}_{2} \mathrm{~N}\right]\right]^{-}\right)$, which has a higher fluorine content. Single-photon near threshold photoionization mass-spectra and photoionization efficiency (PIE) curves, obtained at the Advanced Light Source chemical dynamics beamline in the near threshold region are presented for the cation- as well as anion-substituted IL vapors. In addition, PE spectra are presented, obtained with extreme ultraviolet (EUV) light produced by high-order-harmonic generation. ${ }^{28}$ The combined set of PIE curves and PE spectra data allows extraction of the ionization energies, which are 
attributed to the appearance energies for the dissociative ionization of isolated ion pairs of IL vapors.

\section{Experimental Setup}

The IL vapor source is composed of an aluminum oven body heated by four resistive heater cartridges. The reservoir for the IL is a glass vial, located inside the aluminum oven as shown in Fig. 2. IL samples of $[\mathrm{emim}]\left[\mathrm{Tf}_{2} \mathrm{~N}\right],[\mathrm{dmpim}]\left[\mathrm{Tf}_{2} \mathrm{~N}\right]$ and $\left[\right.$ emim] $\left[\mathrm{Pf}_{2} \mathrm{~N}\right]$ (Sigma, $\geq 97 \%$ purity) are kept under vacuum $\left(\sim 5 \times 10^{-8}\right.$ Torr; $\sim 6.7 \times 10^{-6}$ $\mathrm{Pa}$ ) at $300 \mathrm{~K}$ temperature before the experiment for more than 12 hours in order to remove volatile impurities such as water. When the oven is heated, IL vapor is emitted as an effusive beam through an opening in the oven (Fig. 2). At a $473 \mathrm{~K}$ temperature the vapor pressure of $[\mathrm{emim}]\left[\mathrm{Tf}_{2} \mathrm{~N}\right]$ is estimated to be about $4.3 \times 10^{-4}$ Torr $\left(5.6 \times 10^{-2} \mathrm{~Pa}\right){ }^{25}$ The beam is skimmed by a $1 \mathrm{~mm}$ skimmer before it reaches the interaction region of the ionizing extreme UV beam. The size of the reservoir is $\sim 0.5 \mathrm{ml}$, minimizing fluctuations and/or drifts in the IL vapor source and allowing for continuous 8 hour measurements. Photoions and photoelectrons are analyzed in two different sets of experiments. In one set of experiments, at the chemical dynamics tunable vacuum ultraviolet beamline 9.0.2.3 end-station of the Advanced Light Source (ALS) synchrotron facility, mass spectra of positive ions produced by photoionization of the vapor are measured with a pulsed TOF mass spectrometer. ${ }^{29}$ The mass spectra and ion yield are recorded as a function of wavelength, tunable between $8.2 \mathrm{eV}$ and $9.5 \mathrm{eV}$ in $25 \mathrm{meV}$ steps. Absolute photon energy calibration and monochromator resolution $(\sim 40 \mathrm{meV})$ were determined using the atomic absorption line spectrum of an argon gas filter located between the light source and beamline 9.0.2.3 end-station and used to prevent higher harmonics of the desired photon energies to be transmitted to the end station. At each photon energy setting 500,000 mass-spectra are collected ( 1 minute dwell time) before moving the undulator and monochromator to the next photon energy setting. The scan time is 
intentionally kept much shorter than the typical $~ 9$ hour lifetime of the ALS synchrotron electron beam. The relative photon flux is monitored with a calibrated photodiode and is used to normalize the relative ion yields taken at different photon energies. Photon energy scans were repeated several times (3-8) for each of the IL species. Typical ion yields at $\sim 9.3 \mathrm{eV}$ photon energies are on the order of hundreds up to a few thousands of ions/sec, depending on the photon flux, while the typical background signal at mass peaks of interest was lower than 1 count/sec.

In the other set of experiments, photoelectron spectra are recorded using a $1.3 \mathrm{~m}$ magnetic bottle TOF spectrometer with typical photoelectron energy resolution of $\sim 4 \%{ }^{30}$ The light source used in this set of experiments is a "table top" high order harmonic generation source producing ultrafast extreme UV pulses that are tuned to a fixed $23.2 \mathrm{eV}$ photon energy with a characteristic $70 \mathrm{meV}$ bandwidth. For both experiments, data are recorded as a function of oven temperature. Data taken at a low oven temperature are used for subtraction of background spectrum arising from scattered light and residual gas ionization. The molecular beam of IL vapor recondenses on a collector plate behind the interaction region. The ambient pressure in the interaction vacuum chamber during operation does not exceed $1 \times 10^{-7}$ Torr $\left(1.33 \times 10^{-5} \mathrm{~Pa}\right)$, indicating that the volatilized IL vapor is efficiently recondensed on the skimmer and collector plates that are at $\sim 323 \mathrm{~K}$. The residual IL in the reservoir and the recondensed vapor on the stainless steel plates are examined after each run and do not show any signs of decomposition such as coloration or solid residues. Furthermre, ${ }^{1} \mathrm{H}$ and ${ }^{19} \mathrm{~F}$ NMR analysis that we previously performed on the recondensed $\left[\mathrm{emim}^{-}\left[\mathrm{Tf}_{2} \mathrm{~N}\right]\right.$ liquid, ${ }^{20}$ confirmed that no significant decomposition occurs in our vapor source in agreement with previous studies. ${ }^{21}$ A small, undetectable amount of possible residual impurities or decomposition products are not expected to have an effect on the results presented here. 


\section{$\underline{\text { Results and Discussion }}$}

Figure 3 shows the measured photoelectron binding energy spectra obtained for $[e m i m]\left[\mathrm{Tf}_{2} \mathrm{~N}\right]$ and for the cation and anion substituted $[\mathrm{emim}]\left[\mathrm{Pf}_{2} \mathrm{~N}\right]$ and $[\mathrm{dmpim}]\left[\mathrm{Tf}_{2} \mathrm{~N}\right]$ IL vapors using $23.2 \mathrm{eV}$ light. The overall structure of the PE binding energy spectra do not change drastically, following the small changes in the cation and anion species. Nevertheless, small binding enrgy shifts are evident in the measured spectra. The dashed lines indicate the prominent peak positions of the $[\mathrm{emim}]\left[\mathrm{Tf}_{2} \mathrm{~N}\right]$ PE spectrum at binding energies of $\sim 15 \mathrm{eV}$ and $\sim 16.3 \mathrm{eV}$. It is clear that the corresponding peaks in the $[\mathrm{emim}]\left[\mathrm{Pf}_{2} \mathrm{~N}\right]$ spectrum are shifted towards higher binding energies, while the corresponding peaks in the $[\mathrm{dmpim}]\left[\mathrm{Tf}_{2} \mathrm{~N}\right]$ spectrum are shifted towards lower binding energies. The other two prominent structures in the $[\mathrm{emim}]\left[\mathrm{Tf}_{2} \mathrm{~N}\right]$ PE spectra at about $11.4 \mathrm{eV}$ and $19.7 \mathrm{eV}$ are broader and therefore more difficult to directly compare between the three IL species.

Figure 4 shows a blow up of the normalized PE spectra acquired with $23.2 \mathrm{eV}$ light near the ionization threshold. The error bars (shown in Fig. 3) are omitted for clarity and are on the order of $\sim 0.1$ for $[\operatorname{dmpim}]\left[\mathrm{Tf}_{2} \mathrm{~N}\right]$ and $\sim 0.05$ for $[\operatorname{emim}]\left[\mathrm{Tf}_{2} \mathrm{~N}\right]$ and $[\mathrm{emim}]\left[\mathrm{Pf}_{2} \mathrm{~N}\right]$ on the normalized scale. The solid lines in Fig. 4 show fits of the PE spectra in the near threshold region. We use the simplest threshold function to fit the data, which vanishes below the threshold energy and rises linearly, as $\alpha\left(E-E_{0}\right)$, for binding energies beyond the threshold ionization energy. Here $\mathrm{E}_{0}$ is the fitted threshold energy and $\alpha$ is a fitted overall normalization factor, which depends on the photoionization cross-section, photon flux and sample density. This simple threshold function is convoluted with a $250 \mathrm{meV}$ Gaussian. The width of the Gaussian was also fitted to the data and reflects the combined effect of internal excitation of the hot ion-pairs, the bandwidth of the light source and the experimental response function of the $\mathrm{PE}$ spectrometer. We find that a similar trend of binding enrgy shifts observed for peak positions can be identified in the fitted threshold ionization energies. While for 
$[\mathrm{emim}]\left[\mathrm{Tf}_{2} \mathrm{~N}\right]$, the threshold ionization energy is found to be $8.9 \pm 0.2 \mathrm{eV}$, the threshold for $[\mathrm{emim}]\left[\mathrm{Pf}_{2} \mathrm{~N}\right]$ is shifted to higher binding energies $9.0 \pm 0.2 \mathrm{eV}$, and for $[\mathrm{dmpim}]\left[\mathrm{Tf}_{2} \mathrm{~N}\right]$ the threshold ionization energy is shifted to lower binding energies of $8.7 \pm 0.35 \mathrm{eV}$. The changes in the electronic structure due to the cation and anion substitutions are small and can be mainly characterized by these slight binding enrergy shifts. The main sources of error in this measurement are the limited resolution of the PE spectrometer for fast electrons emitted near the ionization threshold as well as the noise due to background electrons in this PE region. The trends of the small binding energy shifts suggested by the features of the measured PE spectra are reaffirmed in the following by the higher resolution tunable-wavelength PIE spectra measurements.

The photoionization of the IL ion pairs with $23.2 \mathrm{eV}$ photons, which is used to obtain the PE spectra, results in dissociative ionization and a predominant mass peak of the respective intact cation signal in photoionization mass spectra. ${ }^{20}$ With the implementation of tunable wavelengths at the ALS chemical dynamics beamline it is possible to tune the ionizing photon energies near the thresholds of ionization that are revealed by the PE spectra. Thus a relatively soft ionization mechanism is used for examining the stability of isolated IL ion pairs. Figure 5 shows near threshold photoionization mass-spectra of $\left[\operatorname{emim}^{-}\right]\left[\mathrm{Tf}_{2} \mathrm{~N}\right],[\mathrm{emim}]\left[\mathrm{Pf}_{2} \mathrm{~N}\right]$ and $[\mathrm{dmpim}]\left[\mathrm{Tf}_{2} \mathrm{~N}\right] \mathrm{IL}$ vapors, obtained in the near ionization threshold region with photon energies between 8.6 and $9.5 \mathrm{eV}$. Similar to mass spectra obtained with higher photon energies, we find that the predominant positively charged products of the considered ion-pair systems are the respective intact cations, i.e. $[\mathrm{emim}]^{+}$at mass $111 \mathrm{amu}$ and $[\mathrm{dmpim}]^{+}$at mass 139 amu. It is also possible to observe the corresponding ${ }^{13} \mathrm{C}$ isotope peaks at $112 \mathrm{amu}$ and $140 \mathrm{amu}$. The relative yields of the ${ }^{13} \mathrm{C}$ isotope peaks are in agreement with the natural isotope abundance for [emim $]^{+}$with 8 carbon atoms and [dmpim $]^{+}$with 10 carbon atoms. Despite the fact that soft photoionization, with near threshold photon energies, was used no parent mass peaks were observed (not shown). The excellent level of signal-to-noise 
allows us to assess an upper limit of better than $\sim 1: 1000$ for the relative yield of parent ions at the mass of the undissociated ion-pairs, compared to the observed yield of dissociated cation mass. This indicates that the threshold ionization energies of these isolated ion-pairs coincide with the appearance energies of the intact cations produced by dissociative ionization. It is therefore concluded that removal of the highest occupied molecular orbital (HOMO) electron from the IL ion-pairs considered here leads to dissociation and formation of the intact cation.

In order to provide a more precise determination of the small binding energy shifts at the threshold ionization energies, for the cation and anion substitutions, we record PIE curves as a function of photon energy in the near-threshold ionization region. Based on the fact that only dissociative ionization is observed in the ionization threshold region, as identified by PE spectroscopy, we assume that the appearance energy of the intact cation coincides with the ionization energy. Thus, PIE curves are constructed by recording the yield of the respective intact cation signal normalized to the ALS photon flux, as a function of photon energy. Figure 6 shows the near threshold PIE curves, recorded for $[\mathrm{emim}]\left[\mathrm{Tf}_{2} \mathrm{~N}\right],[\mathrm{emim}]\left[\mathrm{Pf}_{2} \mathrm{~N}\right]$ and $[\mathrm{dmpim}]\left[\mathrm{Tf}{ }_{2} \mathrm{~N}\right]$. PIE curves can be compared to the integral of the PE spectra. Therefore we fit the near threshold PIE curves with a function that is the integral of the linear threshold function we successfully used for fitting the near threshold PE spectra, i.e. vanishing yield below the threshold energy and a quadratic rise of the ionization efficiency above the threshold energy. Thus the functional form is $1 / 2 \alpha\left(E-E_{0}\right)^{2}$, where $\alpha$ is a fitted overall normalization factor, $\mathrm{E}$ is the photon energy and $\mathrm{E}_{0}$ is the fitted threshold parameter. Interestingly, this functional form that successfully describes our data is also known as the Fowler law, which was originally developed to describe photoemission from metals, ${ }^{31}$ but was found useful for other systems as well. ${ }^{32}$ Similarly to the PE spectrum fitting procedure, this function is convoluted with a Gaussian resolution function, fitted to be narrower, $50 \mathrm{meV}$, and reflects the better resolution of the monochromator at the 9.0.2.3 beamline. The three 
data sets shown in figure 6 are shown normalized by their fitted overall normalization factor $\alpha$. The solid lines in Fig. 6 are the fitted threshold functions, corresponding to threshold ionization energies of $8.72 \pm 0.03 \mathrm{eV}, 8.78 \pm 0.04 \mathrm{eV}$ and $8.59 \pm 0.03 \mathrm{eV}$ for $[\mathrm{emim}]\left[\mathrm{Tf}_{2} \mathrm{~N}\right],[\mathrm{emim}]\left[\mathrm{Pf}_{2} \mathrm{~N}\right]$ and $[\mathrm{dmpim}]\left[\mathrm{Tf}_{2} \mathrm{~N}\right]$, respectively. The fitted threshold ionization energies are in good agreement with the analysis of the PE spectra within the error bars of the PE spectra analysis. Although within the experimental PE error bars of $0.2 \mathrm{eV}$, the ionization energies obtained by the PE spectra analysis are systematically higher than PIE results, possibly due to a small shift on the order of $0.1 \mathrm{eV}$ in the PE spectrometer calibration. The results reaffirm with higher precision the trends of the small binding energy shifts observed in the PE spectra. It is important to note that for the three IL experiments the reservoir of the ion source is kept at similar temperatures around $500 \mathrm{~K}$, thus the observed shifts are not expected to be due to different molecular beam temperatures. The substitution of the $\left[\mathrm{Tf}_{2} \mathrm{~N}\right]^{-}$anion by $\left[\mathrm{Pf}_{2} \mathrm{~N}\right]^{-}$results in a small $+60 \mathrm{meV}$ shift towards higher electron binding energies. We suggest that this trend may be understood in terms of increased electronegativity due to the substitution of the trifluoromethyl by pentafluoroethyl groups, leading to higher binding energy of the electron to the anion. On the other hand, in the case of $[\mathrm{emim}]^{+}$cation substitution by $[\mathrm{dmpim}]^{+}$, the $-130 \mathrm{meV}$ shift is towards lower binding energies. The addition of the 2 methyl group replacing a hydrogen atom and the replacement of 3-ethyl by the bulkier 3propyl group can act to alter the spacing between the ions, increasing the cation-anion distance, therefore reducing the Coulombic interaction between the cation and the electron of the anion. Thus, we suggest that this particular cation substitution can lead to the observed binding energy shift towards lower binding energies of the anions electron.

\section{Conclusions}

Isolated ion-pairs in the IL vapor of three IL species were studied with a tunable synchrotron light source in the near threshold ionization region, between $8.3 \mathrm{eV}$ and 9.5 $\mathrm{eV}$ photon energies as well as with $23.2 \mathrm{eV}$ photons from a "table-top" high order 
harmonic generation light source. The combined PE spectra, near threshold mass-spectra, and PIE curves allow us to determine ionization threshold energies and to detect small binding energy shifts due to cation and anion substitutions. Theoretical calculations are needed to provide further insights into the electronic structure and the observed binding energy shifts of gas phase IL ion-pairs.

Photoionization of the examined IL vapor predominantly leads to dissociative ionization. Photodetachment of the HOMO electron results in dissociation of the ionized ion-pair system into a neutral fragment and the intact cation that is detected in the massspectra measurements. Previous works using pulsed field ionization of similar IL species have showed that the ionized [cation][anion] $]^{+}$mass peak can be detected in positive ion

mass spectra. ${ }^{27}$ However in the present work even with the introduction of soft photoionization, there is no evidence for the undissociated parent system in the recorded mass-spectra. One possibility is that the parent system formed by photoionization is unstable and spontaneously dissociates on a time scale that is much faster than the typical tens of $\mu$ s between the quasi-continuous ALS light source and the pulsed TOF ion extraction in our experiments.

\section{$\underline{\text { Acknowledgments }}$}

The authors gratefully acknowledge funding from the U.S. Air Force Office of Scientific Research (Grant Nos. FA9550-04-1-0083, F49620-03-1-0212 and FA9300-06C-0023) with additional equipment and support from the Department of Energy under Contract No. DE-AC05CH11231 and the National Science Foundation Extreme Ultraviolet Center, Contract No. EEC-0310717. This work was also supported by the Director, Office of Energy Research, Office of Basic Energy Sciences, Chemical Sciences Division of the U.S. Department of Energy under Contract No. DE-AC02$05 \mathrm{CH} 11231$. 


\section{$\underline{\text { References }}$}

1. Ionic Liquids in Synthesis; P. Wasserscheid,T. Welton, Eds.; Wiley-VCH: Weinheim, 2003.

2. L.A. Blanchard et al., Green processing using ionic liquids and CO2. Nature; 399, 28 (1999).

3. M. Yoshizawa,W. Xu,C.A. Angell, Ionic liquids by proton transfer: Vapor pressure, conductivity, and the relevance of Delta $\mathrm{pK}($ a) from aqueous solutions. Journal of the American Chemical Society; 125, 15411 (2003).

4. M. Susan et al., Bronsted acid-base ionic liquids and their use as new materials for anhydrous proton conductors. Chemical Communications, 938 (2003).

5. R.F. de Souza et al., Room temperature dialkylimidazolium ionic liquid-based fuel cells. Electrochemistry Communications; 5, 728 (2003).

6. J.H. Shin,W.A. Henderson,S. Passerini, Ionic liquids to the rescue? Overcoming the ionic conductivity limitations of polymer electrolytes. Electrochemistry Communications; 5, 1016 (2003).

7. Y.S. Hu et al., Novel room temperature molten salt electrolyte based on LiTFSI and acetamide for lithium batteries. Electrochemistry Communications; 6, 28 (2004).

8. P. Wang et al., Gelation of ionic liquid-based electrolytes with silica nanoparticles for quasi-solid-state dye-sensitized solar cells. Journal of the American Chemical Society; 125, 1166 (2003).

9. P. Wang et al., Charge separation and efficient light energy conversion in sensitized mesoscopic solar cells based on binary ionic liquids. Journal of the American Chemical Society; 127, 6850 (2005).

10. C. Larriba et al., Monoenergetic source of kilodalton ions from Taylor cones of ionic liquids. Journal of Applied Physics; 101, 84303 (2007).

11. P.C. Lozano, Energy properties of an EMI-Im ionic liquid ion source. Journal of Physics D-Applied Physics; 39, 126 (2006). 
12. S. Schneider et al., Ionic liquids as hypergolic fuels. Energy \& Fuels; 22, 2871 (2008).

13. N.V. Plechkova,K.R. Seddon, Applications of ionic liquids in the chemical industry. Chemical Society reviews; 37, 123 (2008).

14. J.L. Anderson,D.W. Armstrong,G.T. Wei, Ionic liquids in analytical chemistry. Analytical Chemistry; 78, 2892 (2006).

15. H. Weingaertner, Understanding ionic liquids at the molecular level: Facts, problems, and controversies. Angewandte Chemie; 47, 654 (2008).

16. I. Krossing,J.M. Slattery, Semi-empirical methods to predict the physical properties of ionic liquids: An overview of recent developments. Zeitschrift Fur Physikalische Chemie-International Journal of Research in Physical Chemistry \& Chemical Physics; 220, 1343 (2006).

17. S. Krischok et al., A comparative study on the electronic structure of the 1-ethyl3-methylimidazolium bis(trifluoromethylsulfonyl)amide $R T$-ionic liquid by electron spectroscopy and first principles calculations. Zeitschrift Fur Physikalische ChemieInternational Journal of Research in Physical Chemistry \& Chemical Physics; 220, 1407 (2006).

18. Z. Meng,A. Dolle,W.R. Carper, Gas phase model of an ionic liquid: semiempirical and ab initio bonding and molecular structure. Journal of Molecular StructureTheochem; 585, 119 (2002).

19. M.J. Earle et al., The distillation and volatility of ionic liquids. Nature; 439, 831 (2006).

20. D. Strasser et al., Photoelectron spectrum of isolated ion-pairs in ionic liquid vapor. journal of physical chemistry A; 111, 3191 (2007).

21. O. Hofft et al., Electronic structure of the surface of the ionic liquid [EMIM][Tf2N] studied by metastable impact electron spectroscopy (MIES), UPS, and XPS. Langmuir; 22, 7120 (2006). 
22. D. Yoshimura et al., Electronic structure of ionic liquids at the surface studied by UV photoemission. Journal of Electron Spectroscopy and Related Phenomena; 144, 319 (2005).

23. J.P. Armstrong et al., Vapourisation of ionic liquids. Physical chemistry chemical physics; 9, 982 (2007).

24. K.R.J. Lovelock et al., Vaporisation of a Dicationic Ionic Liquid. European journal of chemical physics and physical chemistry; 10, 337 (2009).

25. D.H. Zaitsau et al., Experimental vapor pressures of 1-alkyl-3-methylimidazolium bis(trifluoromethylsulfonyl) imides and a correlation scheme for estimation of vaporization enthalpies of ionic liquids. Journal of Physical Chemistry A; 110, 7303 (2006).

26. N. Akai et al., Cryogenic Neon Matrix-isolation FTIR Spectroscopy of Evaporated Ionic Liquids: Geometrical Structure of Cation-Anion 1:1 Pair in the Gas Phase. Journal of Physical Chemistry B; 113, 4756 (2009).

27. J.H. Gross, Molecular Ions of Ionic Liquids in the Gas Phase Journal of the American Society for Mass Spectrometry; 19, 1347 (2008).

28. P.B. Corkum, Plasma Perspective on Strong-Field Multiphoton Ionization. Physical Review Letters; 71, 1994 (1993).

29. L. Belau et al., Vacuum ultraviolet (VUV) photoionization of small water clusters. Journal of Physical Chemistry A; 111, 10075 (2007).

30. L. Nugent-Glandorf et al., A laser-based instrument for the study of ultrafast chemical dynamics by soft $x$-ray-probe photoelectron spectroscopy. Review of Scientific Instruments; 73, 1875 (2002).

31. R.H. Fowler, The analysis of photoelectric sensitivity curves for clean metals at various temperatures. Physical Review; 38, 45 (1931).

32. K. Wong,V.V. Kresin, Photoionization threshold shapes of metal clusters. Journal of Chemical Physics; 118, 7141 (2003). 
Figure Captions:

Figure 1: Schematic drawings of the investigated room temperature IL systems: [1ethyl-3-methylimidazolium][bis(trifluoromethylsulfonyl)imide)] or [emim][Tf $2 \mathrm{~N}]$; 1,2dimethyl-3-propylimidazolium cation substituted $\quad\left[\operatorname{dmpim}_{[}\right]\left[\mathrm{Tf}_{2} \mathrm{~N}\right] ;$ and bis(pentafluoroethylsulfonyl)imide anion substituted $[\mathrm{emim}]\left[\mathrm{Pf}_{2} \mathrm{~N}\right]$.

Figure 2: Schematic view of the IL vapor source and interaction region. The same IL vapor source is used in both experiments. For PE experiments with the high order harmonic generation EUV source, photoelectrons are analyzed by a magnetic bottle TOF spectrometer. For PIE experiments at the 9.0.2.3 ALS beamline, photoions are massanalyzed by a pulsed TOF mass spectrometer as a function of photon energy.

Figure 3: Photoelectron binding energy spectra of IL vapor taken with $23.2 \mathrm{eV}$ photons $(53.7 \mathrm{~nm})$ for $[\mathrm{emim}]\left[\mathrm{Tf}_{2} \mathrm{~N}\right],[\mathrm{emim}]\left[\mathrm{Pf}_{2} \mathrm{~N}\right]$ and $[\mathrm{dmpim}]\left[\mathrm{Tf}_{2} \mathrm{~N}\right]$.

Figure 4: Detail of the PE spectra near the ionization threshold of [emim][Tf $\left.{ }_{2} \mathrm{~N}\right]$, [emim] $\left[\mathrm{Pf}_{2} \mathrm{~N}\right]$ and $[\mathrm{dmpim}]\left[\mathrm{Tf}_{2} \mathrm{~N}\right]$, obtained with $23.2 \mathrm{eV}$ photons $(53.7 \mathrm{~nm})$.

Figure 5: Near-threshold photoionization mass-spectra collected with photon energies in the 8.6 - $9.5 \mathrm{eV}$ range, for (a) $[\mathrm{emim}]\left[\mathrm{Tf}_{2} \mathrm{~N}\right]$, (b) $[\mathrm{emim}]\left[\mathrm{Pf}_{2} \mathrm{~N}\right]$ and (c) $[\mathrm{dmpim}]\left[\mathrm{Tf}_{2} \mathrm{~N}\right]$.

Figure 6: Near-threshold PIE spectra, showing binding energy shifts due to cation and anion substitutions. 


\section{Figure 1}

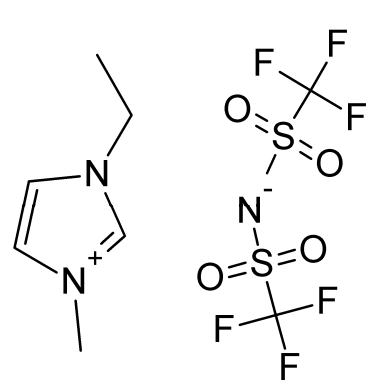

$[\mathrm{emim}]\left[\mathrm{Tf}_{2} \mathrm{~N}\right]$

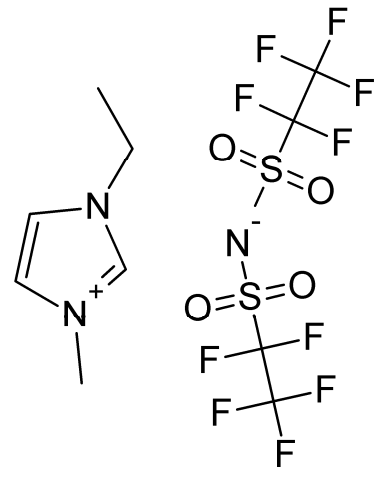

$[\mathrm{emim}]\left[\mathrm{TPf}_{2} \mathrm{~N}\right]$

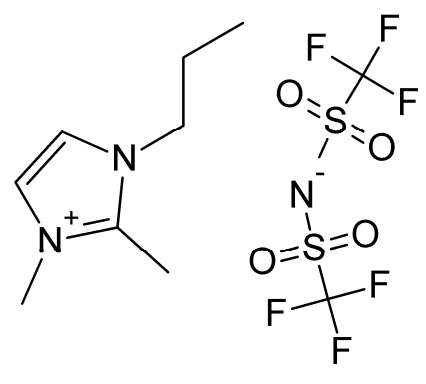

$[\mathrm{dmpim}]\left[\mathrm{Tf}_{2} \mathrm{~N}\right]$

\section{Figure 2}

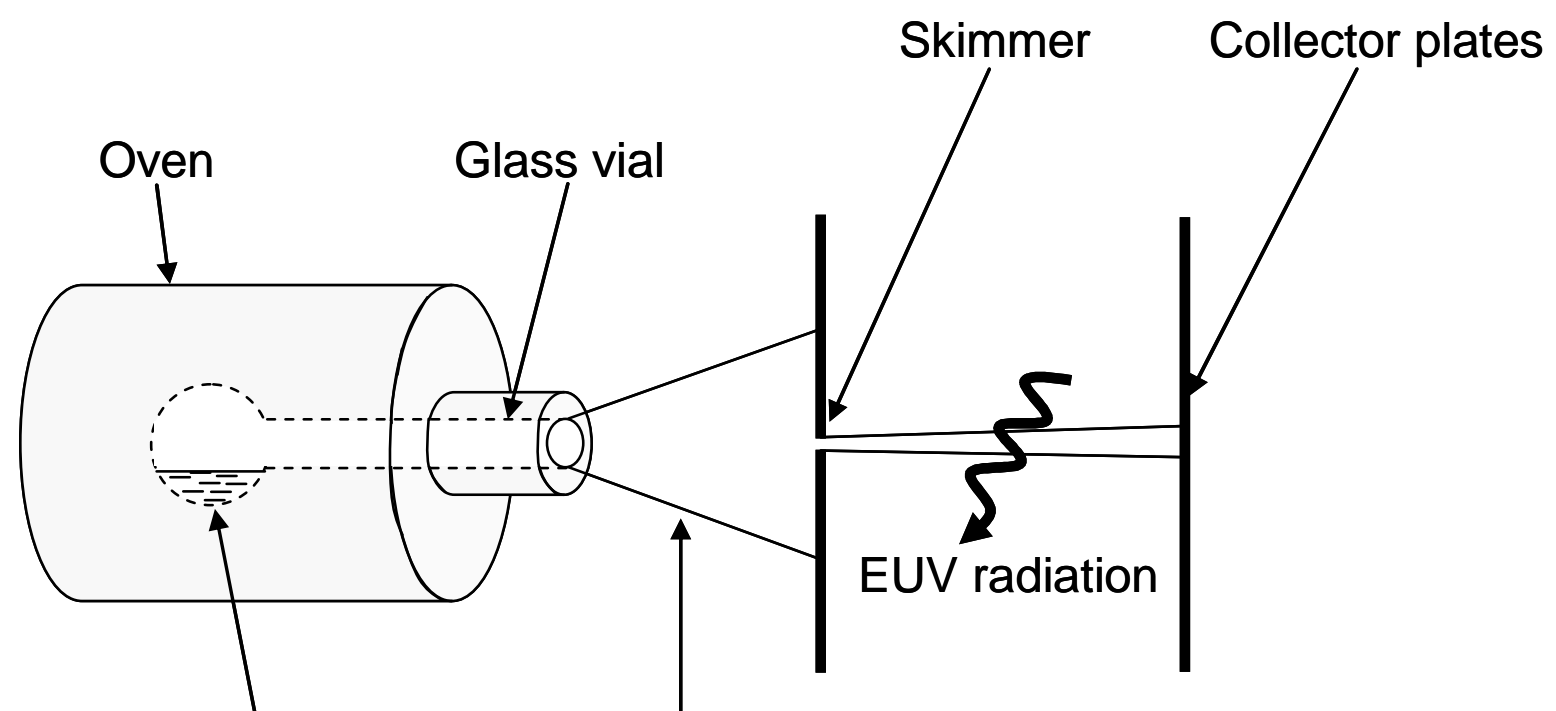

Ionic liquid reservoir Effusive beam 
Figure 3

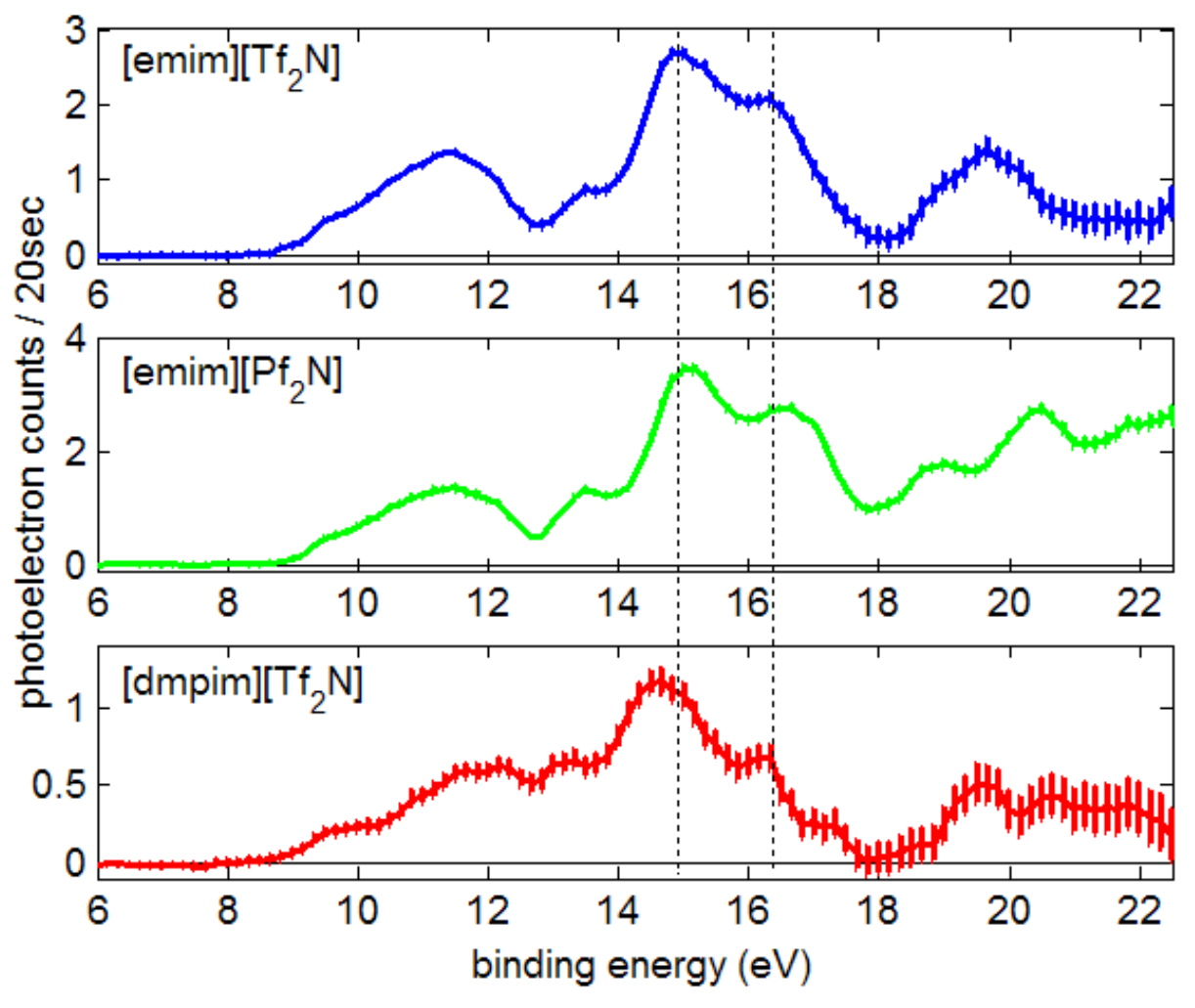

Figure 4

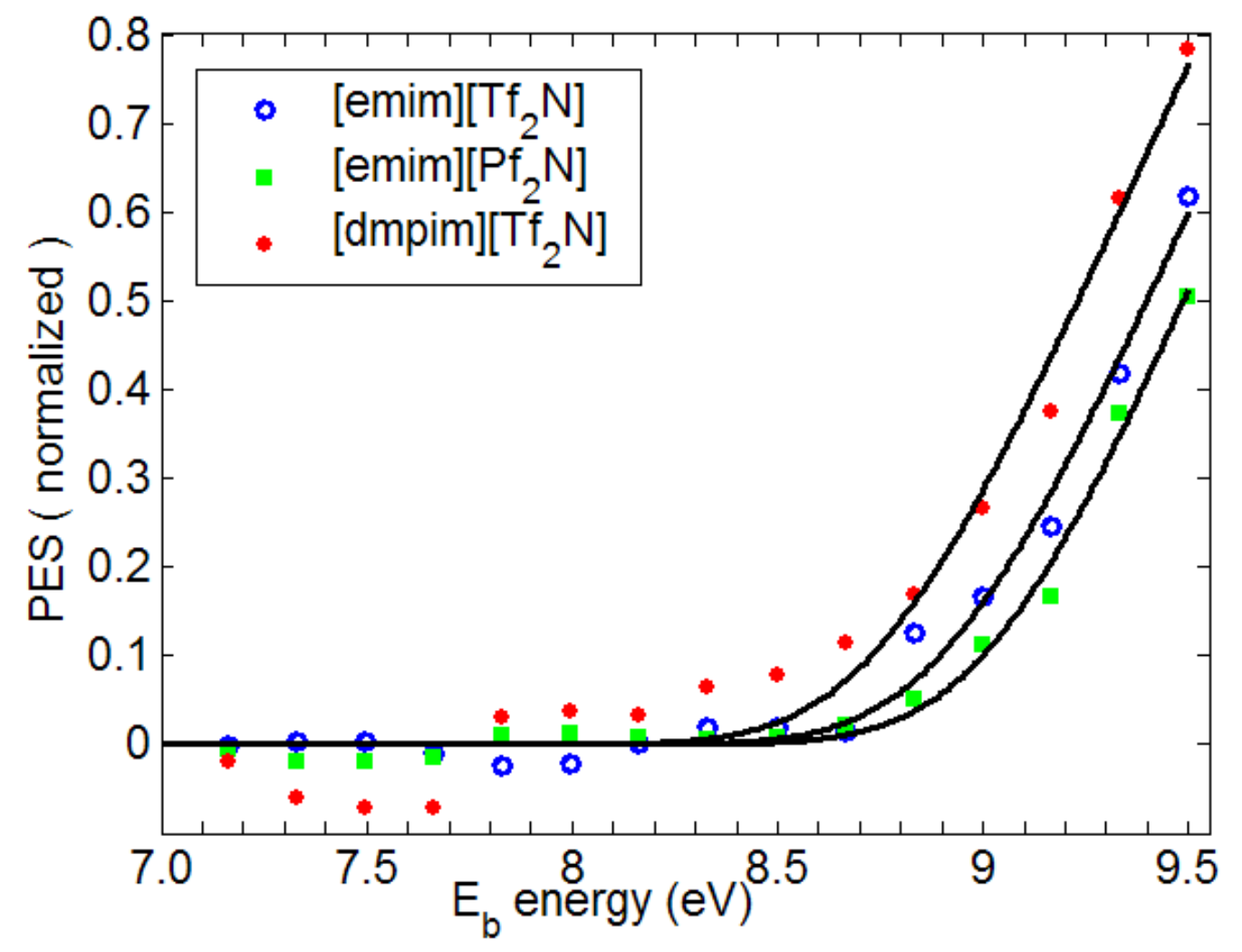




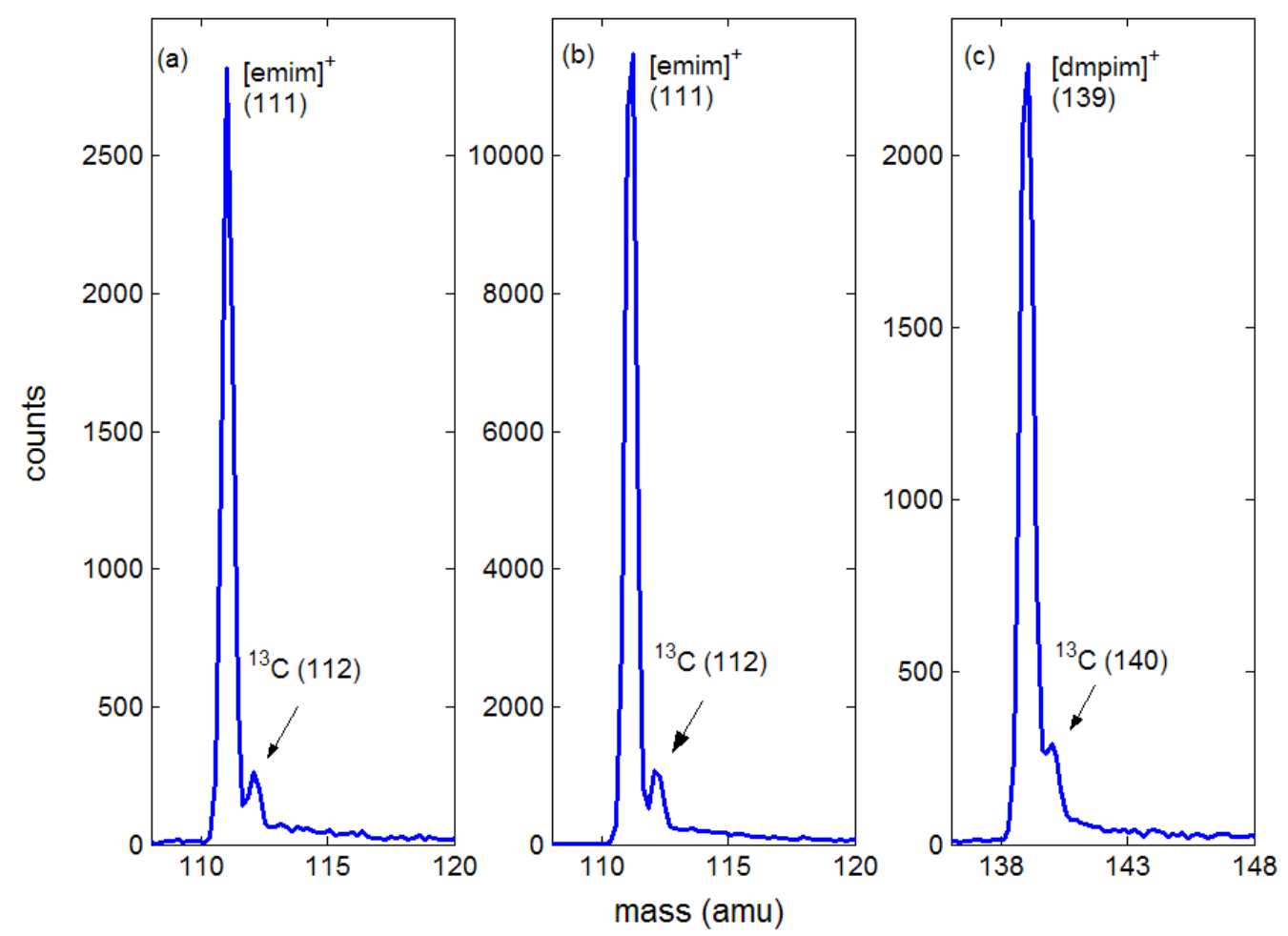

Figure 6

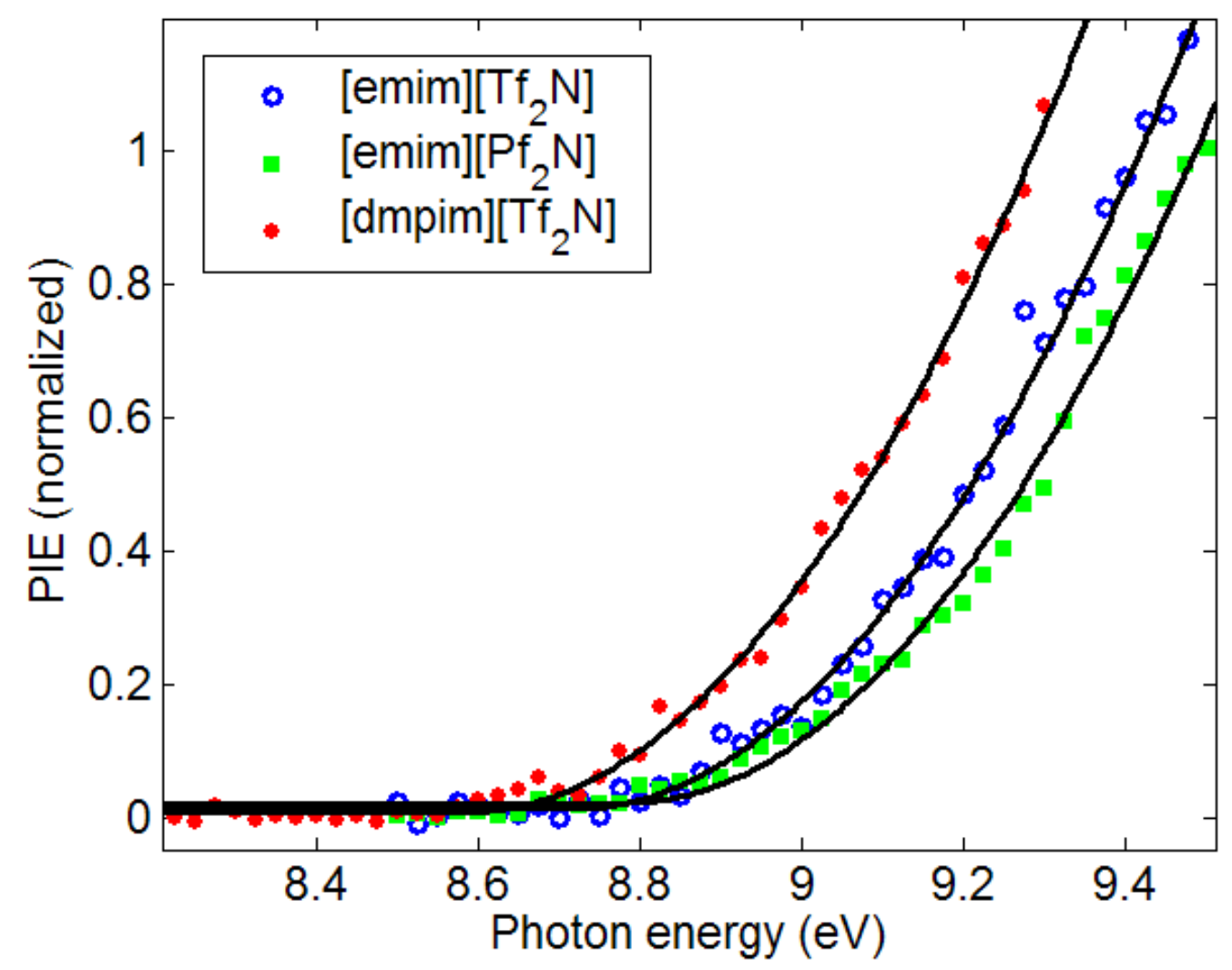

